\title{
Light localization at randomly textured surfaces for solar-cell applications
}

Carsten Rockstuhl, Falk Lederer, Karsten Bittkau, and Reinhard Carius

Citation: Appl. Phys. Lett. 91, 171104 (2007);

View online: https://doi.org/10.1063/1.2800374

View Table of Contents: http://aip.scitation.org/toc/apl/91/17

Published by the American Institute of Physics

\section{Articles you may be interested in}

Local versus global absorption in thin-film solar cells with randomly textured surfaces

Applied Physics Letters 93, 061105 (2008); 10.1063/1.2965117

Angular resolved scattering by a nano-textured $\mathrm{ZnO} /$ silicon interface

Applied Physics Letters 99, 111107 (2011); 10.1063/1.3640238

Engineering the randomness for enhanced absorption in solar cells

Applied Physics Letters 92, 171114 (2008); 10.1063/1.2919094

Optically enhanced amorphous silicon solar cells

Applied Physics Letters 42, 968 (1983); 10.1063/1.93817

Light trapping properties of pyramidally textured surfaces

Journal of Applied Physics 62, 243 (1987); 10.1063/1.339189

Absorption enhancement in solar cells by localized plasmon polaritons

Journal of Applied Physics 104, 123102 (2008); 10.1063/1.3037239

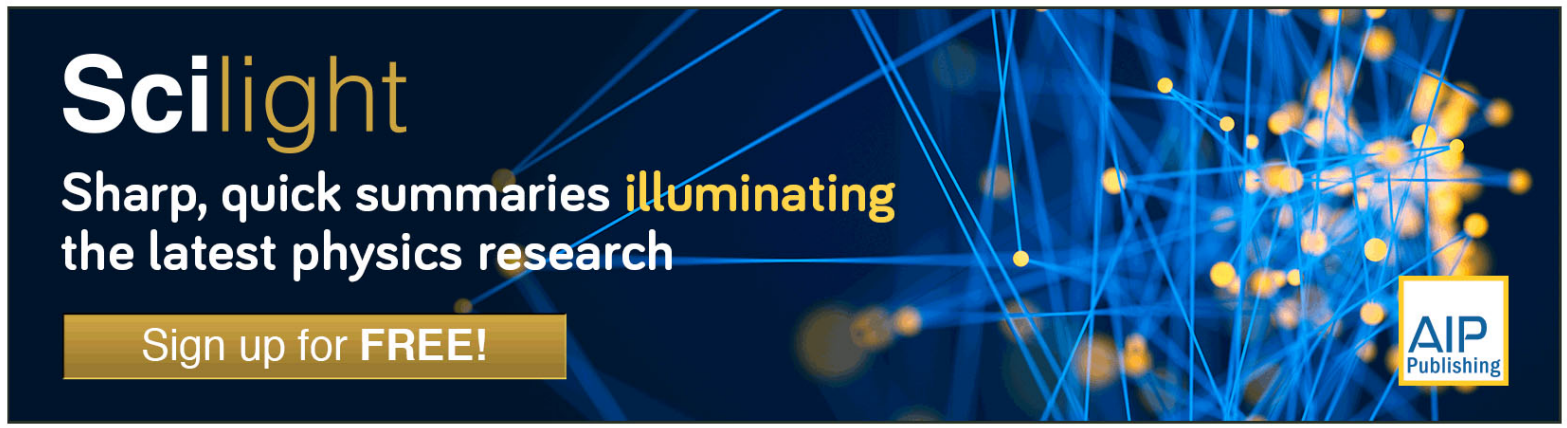




\title{
Light localization at randomly textured surfaces for solar-cell applications
}

\author{
Carsten Rockstuhl ${ }^{\text {a) }}$ and Falk Lederer \\ Institut für Festkörpertheorie und Optik, Friedrich-Schiller-Universität Jena, Max-Wien Platz 1, 07743 \\ Jena, Germany \\ Karsten Bittkau and Reinhard Carius \\ Institut für Energieforschung, Forschungszentrum Jülich GmbH, 52425 Jülich, Germany
}

(Received 8 August 2007; accepted 28 September 2007; published online 23 October 2007)

\begin{abstract}
By using a rigorous diffraction theory, the localization of light near textured zinc oxide $(\mathrm{ZnO})$ surfaces is theoretically investigated and compared with experimental data obtained from scanning-near-field-optical microscopy. Although random by nature, these surfaces show well-defined geometrical features, which cause the formation of localized light patterns near the surface. Particularly, photon jets are observed to emerge from conical surface structures. Because these structures are of primary importance for applications in photovoltaics, we analyze the "real" surface topography of textured $\mathrm{ZnO}$ used in silicon solar cells. With this work, valuable insight is provided into the mechanism of light coupling through randomly textured interfaces. (C) 2007 American Institute of Physics. [DOI: 10.1063/1.2800374]
\end{abstract}

The incorporation of randomly textured surfaces on top of solar cells is usually regarded as a convenient way to reduce reflection losses of light at the interface from air to the solar cell and to increase the scattering of light into the solar cell. ${ }^{1}$ Such surfaces have a promising potential to pave the way toward an efficient photon management in nowadays photovoltaic devices. ${ }^{2}$ In general, the aim of photon management consists in effectively localizing light in the absorbing layer of the solar cell and in reducing losses in other domains by optimizing the involved optical elements.

Typical examples are the deposition of thin film antireflection coatings on top of solar cells, ${ }^{3}$ the incorporation of highly reflecting back sides, ${ }^{4}$ or the use of periodic structures either on top or bottom of the photovoltaic device ${ }^{5}$ However, the intrinsic disadvantage of all periodic structures is the critical dependence of the optical response on wavelength, polarization, and angle of incidence. This allows for an optimized design at particular wavelengths, but a broadband increase of the solar cell's efficiency is hardly achieved. ${ }^{6}$

Such a wavelength dependency is usually not observed for random surfaces. Depending on the ratio of the characteristic feature size of such a surface to the effective wavelength, one usually distinguishes between two operating regimes. If this ratio is much less than unity, light will not resolve the spatial details of the structure and will merely sense an effective graded-index medium. ${ }^{7}$ Such structures allow for a broadband reduction of the Fresnel reflection. For ratios much larger than unity, the structure serves as a light diffuser which shows a Lambertian distribution in the ideal case. The enhancement of intensity inside the solar cell caused by such rough surfaces was shown to be $4 n^{2}{ }^{8}$ They are usually fabricated by etching of the relevant materials. ${ }^{9}$ Regular pyramidal structures are obtained when crystalline silicon substrates are anisotropically etched, e.g., by $\mathrm{KOH}$. Then, the optical response compares to that of rough surfaces with a large ratio of feature size to wavelength.

Although to understand the optical functionality of randomly textured surfaces, these approaches work well within

${ }^{a)}$ Electronic mail: carsten.rockstuhl@uni-jena.de the indicated limits, real structures, barely fit into any of the two regimes. Particularly, the resonance domain, characterized by feature sizes comparable to the wavelength, is not understood so far. In this domain well-established characterization techniques, such as the measurement of the angular distribution of scattered light for identifying the statistical parameters of the structure, are of limited use only. Instead, from a numerical point of view, the solution of the problem asks for a rigorous diffraction theory to account for all relevant effects of light-matter interactions. Only recently, studies on the optical properties of such structures on a subwavelength scale have been performed. ${ }^{10}$ By comparing experimental [scanning near-field optical microscopy (SNOM)] and theoretical results (rigorous diffraction theory that takes into account the real topography), we aim here at bridging the gap between the two indicated limits in understanding the optical functionality of random structures. We concentrate on the optical effects in the near field of the textured structures. This is the important optical domain because thin film solar cells with active layer thicknesses in the range of a few hundred nanometers are essentially near-field devices. It will be shown that such structures have a much richer optical behavior in the resonance domain than usually anticipated. Effects such as light focusing, light localization, and the appearance of photon jets can be identified. The impact of such effects on the design and efficiency of solar cells is discussed. The work here has to be regarded as an initial step toward a full understanding of optical near-field effects in the entire solar cell. This comparison of experimentally observed light fields in the vicinity of randomly textured surfaces with theory may serve as the starting point for further investigations which must include the intensity distribution within the silicon absorber layer and the complete layer stack of the solar cell.

In the present study, randomly textured surfaces were investigated. $\mathrm{ZnO}$ thin films with a thickness of $h$ $=1000 \mathrm{~nm}$ were textured by etching with $\mathrm{HCl}$. Details of the sample preparation can be found in Ref. 10. The structures were characterized by a SNOM with a shear-force feedback loop to measure simultaneously the topography of the 

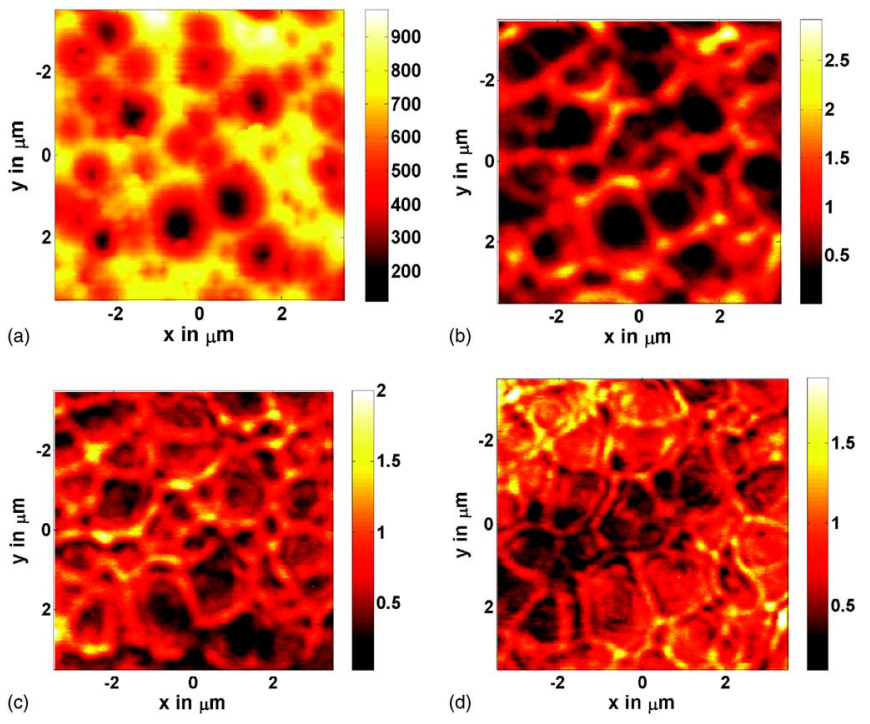

FIG. 1. (Color online) Measured topography in nanometer for a randomly textured surface of $\mathrm{ZnO}$ on top of a quartz substrate (a). The near-field intensity in arbitrary units slightly above this textured surface as measured with a scanning near-field optical microscope is shown for wavelengths of $780 \mathrm{~nm}$ (b), $658 \mathrm{~nm}$ (c), and $488 \mathrm{~nm}$ (d), respectively.

sample and the electromagnetic near field. The SNOM fiber tip provides a spatial resolution of less than $100 \mathrm{~nm}$. The measured signal is regarded as being proportional to the light intensity at the tip position. The instrument is fed with coherent light from different sources ranging from 488 to $780 \mathrm{~nm}$ to detect the optical near field at well-defined wavelengths covering the spectral range of an $a-\mathrm{Si}: \mathrm{H}$ solar cell.

Figure 1(a) shows a measured topology for a representative sample over an area of $7 \times 7 \mu \mathrm{m}^{2}$. It can be seen that, although random in nature, the structure consists of welldefined circular craters with variable size. Occasionally, the craters are almost touching, causing the occurrence of elongated rims. The typical diameter of the craters was found to be $800-1000 \mathrm{~nm}$. The largest depth was measured to be approximately $1000 \mathrm{~nm}$. Thus we conclude that the $\mathrm{ZnO}$ thin films were etched almost down to the substrate.

Measured SNOM images, taken from the same surface at the representative wavelengths of 780,658 , and $488 \mathrm{~nm}$, are shown in Figs. 1(b)-1(d), respectively. The fields were monitored at a constant distance of $20 \mathrm{~nm}$ above the surface. The structure was illuminated from the back through the substrate and the transmitted fields were measured; the reverse configuration yielded similar results.

To compare results with simulations, we applied the finite-difference time-domain method to rigorously calculate the electromagnetic near field. ${ }^{11}$ In the simulation, the topology of the $7 \times 7 \mu \mathrm{m}^{2}$ surface as measured by the feedback loop of the SNOM was fully taken into account to permit for a direct comparison. The lowest point of the surface was assumed to be in contact with the substrate and the measured height profile corresponds to the terminating $\mathrm{ZnO}$ surface. In the simulations, the refractive index of the substrate and of the $\mathrm{ZnO}$ layer were $n_{\mathrm{SiO} 2}=1.5$ and $n_{\mathrm{ZnO}}=1.94$, respectively. The structure was discretized with $\Delta x=\Delta y=\Delta z=15 \mathrm{~nm}$ and illuminated by an $x$-polarized plane wave from the substrate. No pronounced polarization sensitivity was found. The boundaries of the computation window in propagation direction ( $z$ axis) were chosen to be perfectly matched layers. In
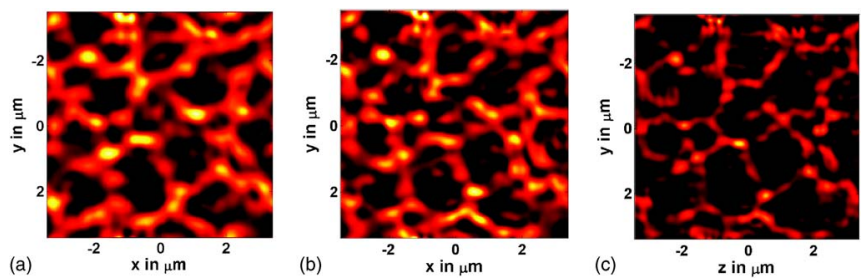

FIG. 2. (Color online) Simulated intensity of $200 \mathrm{~nm}$ above the randomly textured $\mathrm{ZnO}$ surface at wavelengths of $780 \mathrm{~nm} \mathrm{(a),} 658 \mathrm{~nm}$ (b), and $488 \mathrm{~nm}(\mathrm{c})$. Black and white colors denote zero and maximum intensity.

the $x$ - and $y$-directions, periodic boundaries were assumed. The artificially introduced periodicity did not affect the calculated field at all, as deduced from simulations at a reduced sample size. This showed that all observed effects are not associated with a particular feature size that periodically repeats along the surface. Instead, the scattering properties of the individual elements forming the surface and their interference with fields emerging from the adjacent elements are of primary importance. To compare measurements with simulations, we calculated the intensity (defined here as $E_{x}^{2}$ $\left.+E_{y}^{2}+E_{z}^{2}\right)$ to be $200 \mathrm{~nm}$ above the surface at all positions $(x, y)$. Good qualitative agreement between measurement and simulation was found. Although the tip-surface distance in the measurement was only about $20 \mathrm{~nm}$ best agreement was found for the chosen surface distance of $200 \mathrm{~nm}$ in the simulation. A possible explanation might be the transfer function of the SNOM tip, which acts like a low-pass filter with a finite transverse cutoff wave number. The shorter exponential decay length of evanescent amplitudes with larger wave numbers in free space equals in its cause the low-pass filter of the SNOM transfer function, hence allowing us to compare theoretical and experimental results. Furthermore, we cannot rule out the possibility that the presence of the SNOM tip might deteriorate slightly the measured field distribution. As the agreement between measurement and simulation is always found to be good even for strongly different features, we conclude that the tip-sample interaction might only cause the deviation of details in the measured field distribution as compared to the real one.

Starting with the longest wavelength of $780 \mathrm{~nm}$ [measurement is shown in Fig. 1(b), simulation in Fig. 2(a)] we observe an intensity enhancement along the rims of the crater with rather weak field localization normal to the rims. No localization shows up parallel to the rims. The field is stronger localized in both transverse directions where rims are crossing and forming a well-defined maximum at the surface with a conical shape. The appearance of localized light at well-defined sites randomly distributed over the surface is stronger pronounced at shorter wavelengths. The results at $658 \mathrm{~nm}$ [measurement is shown in Fig. 1(c), simulation in Fig. 2(b)] show that the points of localization remain nearly the same and the spot size is reduced, as expected for a shorter wavelength. At the shortest wavelength, the measurement is shown in Fig. 1(d), the intensity is slightly distorted due to a fringe like pattern. From simulations, however, we can see in Fig. 2(c) that the effect of light localization is further pronounced and light concentrates in narrow spatial domains above the structure.

This light localization might be explained by the concurrence of two effects. The first one is the lightning rod effect. It causes the enhancement of near fields close to sites where 

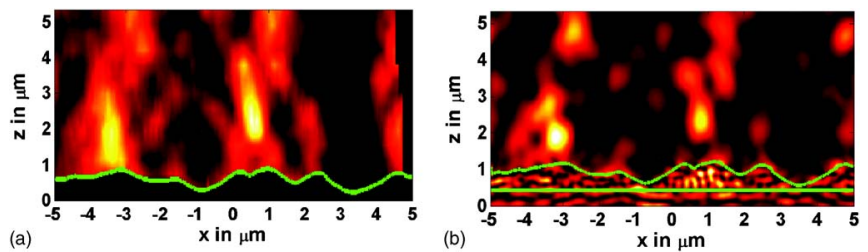

FIG. 3. (Color online) Measured (a) and simulated intensities (b) in a cross section of the field distribution above a random surface for a wavelength of $780 \mathrm{~nm}$ for the illuminating wave field. The light propagation is in the positive $z$ direction through the substrate. Black and white colors denote zero and maximum intensity, respectively.

the surface curvature of micro- and nanometer sized particles is the largest. The intensity in the apex is material independent and merely a function of geometry. For example, at particles with an elongated hemispheroidal shape, the enhancement is approximately $(a / b)^{2} / \ln (a / b) \gg 1$, with $a$ and $b$ being the radii of the two relevant axes. ${ }^{12}$ The third axis $c$ is equal to $b$. This light localization is restricted to the near field of the particles. The origin of the effect compares to the formation of localized plasmon polaritons excited in nanoparticles made of noble metals. The second effect that contributes to the light concentration is a focusing effect by the surface profile that has, at least locally, a preferentially parabolic- or coniclike profile for points were localization was observed. Light concentration caused by focusing should preferentially occur at a certain distance from the surface. This effect is a result of constructive interference of light emerging from the surface profile with an appropriate phase delay.

Because measurements in a single plane behind the structure did not allow to elucidate which effect governs the response of the textured structure, we proceeded in measuring the intensity distribution in various planes parallel to the surface normal. This allows for monitoring the threedimensional intensity distribution. Exemplarily, in Fig. 3(a), the intensity is shown at a wavelength of $780 \mathrm{~nm}$ in the plane with $y=1 \mu \mathrm{m}$ [compare to the topography image in Fig. 1(a)]. The measurement is compared with theoretical simulations shown in Fig. 3(b). Here, the field in $z$ direction was measured with a spatial resolution of $350 \mathrm{~nm}$. In agreement with the low-pass filtering response of the SNOM tip transfer function, the measured intensity is slightly smeared out and the fine details that appear in the simulation are experimentally not resolved. Nevertheless, all qualitative features do appear nicely in both experiment and simulation, namely, light localization close to the surface and light focusing further away from the surface. Combining both effects, we observe for these samples the appearance of photon jets emerging from high curvature points of the surface, causing rather elongated light strings in the air. The light localization that occurs above the surface at $x=-3 \mu \mathrm{m}$, is caused by a strong curvature of the surface in $y$ direction and is, therefore not directly deducible from the surface profile. As the light localizes rather close to the surface, we conclude that this concentration is dominated by the lightning rod effect. It occurs if the surface profile is sufficiently strong curved, independent from the macroscopic extension of this geometrical feature. On the contrary, the light localized above the surface at $x=1 \mu \mathrm{m}$ focuses further away from the surface, hence the effect of light focusing dominantly contributes to the observed light localization.
In the simulation, we can additionally observe light localization inside the $\mathrm{ZnO}$ material below points of a high curvature, experimentally not accessible. Particularly, for the present sample, such localization is observed in the $\mathrm{ZnO}$ film at $x=1 \mu \mathrm{m}$. This is in agreement with the observation that there light localization is dominated by a focusing effect due to the strong curvature. The back reflected light at the interface from $\mathrm{ZnO}$ to air interferes constructively inside the material.

The present structure can be incorporated into solar cell devices to enhance the absorption. For structures in the resonance domain the light localization near such randomly textured surfaces causes an enhancement of the near-field amplitude, which will enhance the absorption. Similar observations were made for metallic nanoparticles incorporated in solar cells, although there the light localization is caused by localized plasmon polaritons. ${ }^{13}$ The advantage of using rough surfaces in high permittivity materials as compared to metallic nanoparticles is their much lower absorbance of light. This absorbance is detrimental to the performance of solar cells. Furthermore, the localization of light at surfaces with large curvatures is less sensitive to the wavelength, as shown here experimentally and theoretically.

To summarize, light localization in the near field of randomly textured surfaces was analyzed experimentally and theoretically. Typical feature sizes of the present structures are comparable to the wavelength. The origin of the observed localization was identified to be the simultaneous appearance of the lightning rod effect and focusing of light due to the strong curvature of the surface. Similar structures as studied here show great promise for thin film solar cell applications. However, to fully understand the functionality of randomly textured surfaces further studies have to be conducted. Most importantly, the connection between the possibility to localize light by such surfaces and the resulting absorption in a deposited Si layer has to be understood. This will allow us to optimize the texture of the surface.

We acknowledge the partial financial support of this work by the Deutsche Forschungsgemeinschaft (PAK88) and the Federal Ministry of Education and Research (Nanovolt). Furthermore, we thank J. Hüpkes for providing us with the samples for this study. Parts of computations were performed on the IBM p690 cluster JUMP of the JvN Forschungszentrum Jülich, Germany.

${ }^{1}$ M. A. Green, Prog. Photovoltaics 10, 235 (2002).

${ }^{2}$ J. Nelson, The Physics of Solar Cells (Imperial College, London, 2003).

${ }^{3}$ J. Zhao, A. Wang, P. Altermatt, and M. A. Green, Appl. Phys. Lett. 66, 3636 (1995).

${ }^{4}$ A. Banerjee and S. Guha, J. Appl. Phys. 69, 1030 (1991).

${ }^{5}$ P. Sheng, A. N. Bloch, and R. S. Stepleman, Appl. Phys. Lett. 43, 579 (1983).

${ }^{6}$ C. Haase and H. Stiebig, Prog. Photovoltaics 14, 629 (2006).

${ }^{7}$ D. E. Aspnes, J. B. Theeten, and F. Hottier, Phys. Rev. B 20, 3292 (1979).

${ }^{8}$ E. Yablonovich, J. Opt. Soc. Am. 72, 899 (1982).

${ }^{9}$ E. Yablonovitch and G. D. Cody, IEEE Trans. Electron Devices 29, 300 (1982).

${ }^{10}$ K. Bittkau, C. Lienau, and R. Carius, Phys. Rev. B 76, 035330 (2007).

${ }^{11}$ A. Farjadpour, D. Roundy, A. Rodriguez, M. Ibanescu, P. Bermel, J. D. Joannopoulos, S. G. Johnson, and G. Burr, Opt. Lett. 31, 2972 (2006).

${ }^{12}$ G. T. Boyd, T. Rasing, J. R. R. Leite, and Y. R. Shen, Phys. Rev. B 30, 519 (1984).

${ }^{13}$ S. Pillai, K. R. Catchpole, T. Trupke, and M. A. Green, J. Appl. Phys. 101, 093105 (2007). 